\title{
MEAN REVERSION IN THE US TREASURY CONSTANT MATURITY RATES
}

\author{
Guglielmo Maria Caporale ${ }^{\mathrm{a}}$ \\ Luis A. Gil-Alanab \\ ${ }^{\text {a }}$ Brunel University, London \\ ${ }^{\mathrm{b}}$ University of Navarra
}

\section{February 2007}

\begin{abstract}
The daily structure of the US Treasury Constant Maturity Rates is investigated in this paper by means of fractional integration techniques. Using a version of the tests of Robinson (1994) along with a model selection criterion based on diagnostic tests on the residuals, we show that the behaviour of this series can be captured by I(d) statistical models with the fractional parameter d close to, but smaller than 1 , which indicates mean reversion.
\end{abstract}

Keywords: Fractional integration; Long memory.

JEL Classification: C22, G14

Corresponding author: Professor Guglielmo Maria Caporale, Brunel University, Uxbridge, Middlesex UB8 3PH, UK. Tel.: +44 (0)1895 266713. Fax: +44 (0)1895 269770. Email: Guglielmo-Maria.Caporale@brunel.ac.uk

The second-named author gratefully acknowledges financial support from the Minsterio de Ciencia y Tecnologia, (SEJ2005-07657/ECON), Spain. 


\section{Introduction}

Adequately modelling macroeconomic and financial time series is crucial for understanding the economy. However, there exists little consensus about the best way to describe their stochastic behaviour. In the last couple of decades unit root models, based on first differences of the data, have become very popular, especially since the seminal work of Nelson and Plosser (1982), who, following on from Box and Jenkins (1970), showed that many US macroeconomic series can be specified in terms of unit roots. Their short-run (stationary) dynamic behaviour is also of interest. Consider, for instance, financial series: if they follow a random walk process, they are completely unpredictable, whilst weak dependence implies that there is a predictable component. This paper is concerned with both their long- and short-run components. We show first that they can be specified in terms of I(d) statistical models, which include the unit root as a particular case when $d=1$. A model selection criterion is then adopted to choose the correct model specification for the short-run dynamics. The structure of the paper is as follows: Section 2 briefly describes a version of the tests of Robinson (1994) which is suitable to test I(d) statistical models in the context of weakly autocorrelated disturbances. In Section 3, the tests are applied to the US Treasury Constant Maturity Rates. Section 4 proposes a model selection criterion based on diagnostic tests on the residuals in order to determine the best model specification for the series. Section 5 analyses impulse responses of the selected models, while Section 6 concludes.

\section{Testing of $\mathbf{I}(\mathrm{d})$ hypotheses}

A simple version of the tests of Robinson (1994) consists in testing the null hypothesis:

$$
H_{o}: d=d_{o} \text {, }
$$

in a model given by:

$$
(1-L)^{d} x_{t}=u_{t}, \quad t=1,2, \ldots,
$$


where $\mathrm{x}_{\mathrm{t}}$ is the time series we observe; $\mathrm{u}_{\mathrm{t}}$ is an $\mathrm{I}(0)$ process, defined as a covariance stationary process with spectral density function that is positive and finite at the zero frequency, and $\mathrm{d}_{\mathrm{o}}$ in (1) can be any real number. The test statistic, which is based on the Lagrange Multiplier (LM) principle, is then given by:

$$
\hat{r}=\left(\frac{T}{\hat{A}}\right)^{1 / 2} \frac{\hat{a}}{\hat{\sigma}^{2}},
$$

where $\mathrm{T}$ is the sample size, and

$$
\begin{gathered}
\hat{a}=\frac{-2 \pi}{T} \sum_{j=1}^{T-1} \psi\left(\lambda_{j}\right) g\left(\lambda_{j} ; \hat{\tau}\right)^{-1} I\left(\lambda_{j}\right) ; \quad \hat{\sigma}^{2}=\frac{2 \pi}{T} \sum_{j=1}^{T-1} g\left(\lambda_{j} ; \hat{\tau}\right)^{-1} I\left(\lambda_{j}\right) ; \\
\hat{A}=\frac{2}{T}\left(\sum_{j=1}^{T-1} \psi\left(\lambda_{j}\right)^{2}-\sum_{j=1}^{T-1} \psi\left(\lambda_{j}\right) \hat{\varepsilon}\left(\lambda_{j}\right)^{\prime} \times\left(\sum_{j=1}^{T-1} \hat{\varepsilon}\left(\lambda_{j}\right) \hat{\varepsilon}\left(\lambda_{j}\right)^{\prime}\right)^{-1} \times \sum_{j=1}^{T-1} \hat{\varepsilon}\left(\lambda_{j}\right) \psi\left(\lambda_{j}\right)\right) \\
\psi\left(\lambda_{j}\right)=\log \left|2 \sin \frac{\lambda_{j}}{2}\right| ; \quad \hat{\varepsilon}\left(\lambda_{j}\right)=\frac{\partial}{\partial \tau} \log g\left(\lambda_{j} ; \hat{\tau}\right) ; \quad \lambda_{j}=\frac{2 \pi j}{T} .
\end{gathered}
$$

$\mathrm{I}\left(\lambda_{\mathrm{j}}\right)$ is the periodogram of $\hat{u}_{t}=(1-L)^{d_{o}} y_{t}$ and g above is a known function coming from the spectral density of $\mathrm{u}_{\mathrm{t}}$, (e.g. $\mathrm{g} \equiv 1$ if $\mathrm{u}_{\mathrm{t}}$ is white noise).

Based on $\mathrm{H}_{\mathrm{o}}$ (1), Robinson (1994) established that under certain regularity conditions,

$$
\hat{r} \rightarrow_{d} N(0,1) \text { as } T \rightarrow \infty \text {. }
$$

Thus, an approximate $100 \alpha \%$ level test of (1) will reject $H_{o}$ against the alternative: $H_{a}: d>d_{o}$ (d $\left.<\mathrm{d}_{\mathrm{o}}\right)$ if $\hat{r}>\mathrm{z}_{\alpha}\left(\hat{r}<-\mathrm{z}_{\alpha}\right)$, where the probability that a standard normal variate exceeds $\mathrm{z}_{\alpha}$ is $\alpha$. He also showed that the tests are efficient in the Pitman sense, i.e., that against local alternatives of the form: $\mathrm{H}_{\mathrm{a}}: \mathrm{d}=\mathrm{d}_{\mathrm{o}}+\delta \mathrm{T}^{-1 / 2}$, with $\delta \neq 0, \hat{r}$ has a limit normal distribution with variance 1 and mean that cannot (when $\mathrm{u}_{\mathrm{t}}$ is Gaussian) be exceeded in absolute value by that of any rival regular statistic. This version of the tests of Robinson (1994) was used in empirical applications in Gil-Alana and Robinson (1997) and Gil-Alana (2000), and other applied studies 
of the tests based on seasonal (quarterly and monthly) and cyclical models are Gil-Alana and Robinson (2001) and Gil-Alana (1999, 2001) respectively.

\section{Using I(d) models for a US financial series}

The time series analysed in this section is the daily structure of the $1,3,5,7$ and 10 year US Treasury Constant Maturity Rates (denoted by D1, D3, D5, D7 and D10 respectively), obtained from the H.15 Release of the Federal Reserve Board of Governors. The starting date is 3 January 2000 and all series end on 23 February 2001.

Denoting each series in turn $x_{t}$, we employ throughout model (2), testing $H_{0}$ (1) for values $d_{0}=0.50,(0.10), 1.50$, with white noise and weakly autocorrelated disturbances. The test statistic reported in Table 1 is the one-sided one given by $\hat{r}$ in (3). Thus, for a given $d_{0}$, significantly positive values of $\hat{r}$ are consistent with an order of integration higher than that, whereas significantly negative ones are consistent with smaller orders of integration. In view of this, we should expect a monotonic decrease in the value of the test statistic with respect to $d_{0}$, and this is precisely what is shown in Table 1, which presents the results based on white noise, $\mathrm{AR}(1)$ and $\mathrm{AR}(2)$ disturbances.

\section{(Table 1 about here)}

A notable feature which is apparent in Table 1(i) (with white noise $\mathrm{u}_{\mathrm{t}}$ ) is the fact that the only non-rejection values occur for all series when $d=1$, implying that the series can be specified as random walk processes. However, these results might reflect to a large extent unaccounted $\mathrm{I}(0)$ autocorrelation in $\mathrm{u}_{\mathrm{t}}$. Therefore, in Tables 1 (ii) and (iii), we allow the disturbances to follow AR(1) and AR(2) processes respectively. Higher order autoregressions were also considered, and the results were very similar to those reported in the table.

When imposing $\operatorname{AR}(1) \mathrm{u}_{\mathrm{t}}$, we find that the unit root null hypothesis cannot be rejected for any series. Non-rejections also occur when $d=0.90$ and 1.10 for D1 and D3; when $d=0.90$ 
for D5; and when $\mathrm{d}=0.80$ and 0.90 for D7 and D10. Thus, it seems that the order of integration is slightly smaller as the number of years in the maturity rate increases. Similarly, if we allow $\mathrm{AR}(2)$ disturbances, the unit root case cannot be rejected for any series, though orders of integration slightly smaller may also be plausible in some cases, especially for D5, D7 and D10. To sum up, if we impose white noise disturbances, a random walk model cannot be rejected for these series, however, when allowing weakly parametrically autocorrelated disturbances, both unit roots and orders of integration slightly smaller than or greater than one appear to be suitable specifications for describing the nonstationary behaviour of the series. The distinction between $\mathrm{I}(\mathrm{d})$ models with d smaller than or greater than one is important from an economic viewpoint. Specifically, a time series which is $\mathrm{I}(\mathrm{d})$ with $\mathrm{d} \geq 1$ will be nonstationary and non- mean-reverting, with the effects of shocks persisting forever. On the other hand, if $d<1$, the series will be nonstationary (if $d \geq 0.50$ ) but mean-reverting, with the effects of shocks dying away in the long run. Consequently, it is crucial to determine correctly the order of integration of the series. In the following section, a model selection criterion is adopted to determine the best model specification for each of the series analysed here.

\section{A model selection criterion}

As a first step, we investigate more in depth the order of integration of the series of interest. Specifically, we redo the calculations reported in Table 1 , but this time allowing $\mathrm{d}_{\mathrm{o}}$ to take values $0.50(0.01)$, 1.50, choosing for each series the value which produces the lowest $|\hat{r}|$ across $d_{0}$. The intuition behind this procedure is that the selected model will be the one with the closest residuals to a white noise process. We repeat this for white noise, AR(1) and AR (2) disturbances; the results are displayed in Table 2.

(Table 2 about here) 
It can be seen that in all cases except one (corresponding to D10 with $A R(2) u_{t}$ ), the orders of integration are smaller than or equal to 1. Also, in two cases (D5 and D7 again with AR(2) disturbances), this value is exactly 1 , while for the remaining models $d$ oscillates between 0.88 and 0.98 .

\section{(Table 3 about here)}

Next, we examine each of these potential models by looking at several diagnostic tests on the residuals. In particular, we perform tests of no serial correlation, homocedasticity and functional form. The results are given in Table 3. Starting with D1, models B and C both pass the diagnostic tests at the 95\% significance level (though in the case of model C the second AR coefficient is not significant). For D3 and D5 the best specifications are those with AR(1) disturbances (model B), while D7 and D10 can best be described in terms of pure I(d) processes with no weak dependence (model A). In all these cases $d$ is smaller than 1 , implying that mean reversion occurs; D1, D3 and D5 have a component of weak dependence, whilst D7 and D10 are specified with white noise disturbances.

\section{Impulse response functions}

The impulse responses of each of the selected models are shown in Table 4. As one would expect, in view of the large values for the fractional differencing parameters, the series are highly persistent; however, the fact that $\mathrm{d}$ is in all cases smaller than 1 implies that mean reversion occurs, with the effects of shocks dying away in the long run.

\section{(Table 4 about here)}

D1 and D3 are the most persistent series, with an increasing initial effect that is above 1 even 100 periods after the initial shock in case of D1, and six periods after in the case of D3. For the latter series (and also for both D7 and D10, whose behaviour can described by the same 
model), more than $80 \%$ of the initial shock is still affecting the series after 200 periods, while D5 appears to be less persistent, with the effect of shocks dying away much quicker.

\section{Conclusions}

In this paper we have analysed the daily structure of the US 1- 3- 5- 7- and 10-year Treasury Constant Maturity Rates by means of fractional integration techniques. In particular, we have used a testing procedure due to Robinson (1994), which, unlike other methods, has standard (normal) null and local limit distributions. The initial results indicated that, if the disturbances are white noise, the series can be modelled as random walk processes. However, when allowing for weakly parametrically autocorrelated disturbances, fractional orders of integration, with d smaller than or greater than one, are also plausible. A model selection criterion, based on diagnostic tests on the residuals, was then adopted to determine the best model specification in each case. It suggested that for all series the best statistical model is an I(d) one, with d close

to but smaller than 1 . Therefore, these series appear to be mean reverting, with the effects of shocks disappearing in the long run. 


\section{References}

Box, G.E.P. and G.M. Jenkins, 1970, Time series analysis, Forecasting and control, Holden Day, San Francisco. CA.

Gil-Alana, L.A., 1999, Testing fractional integration with monthly data, Economic Modelling 16, 613-629.

Gil-Alana, L.A., 2000, Mean reversion in the real exchange rates, Economics Letters 69, 285288.

Gil-Alana, L.A., 2001, Testing stochastic cycles in macroeconomic time series, Journal of Time Series Analysis 22, 411-430.

Gil-Alana, L.A. and P.M. Robinson, 1997, Testing of unit roots and other nonstationary hypotheses in macroeconomic time series, Journal of Econometrics 80, 241-268.

Gil-Alana, L.A. and P.M. Robinson, 2001, Seasonal fractional integration in the UK and Japanese consumption and income, Journal of Applied Econometrics 16, 95-114.

Nelson, C.R., and C.I. Plosser, 1982, Trends and random walks in macroeconomic time series, Journal of Monetary Economics 10, 139-162.

Robinson, P.M., 1994, Efficient tests of nonstationary hypotheses, Journal of the American Statistical Association 89, 1420-1437. 
TABLE 1

Testing $H_{0}(1)$ in (2) with the tests of Robinson (1994)

i) White noise disturbances

\begin{tabular}{|c|c|c|c|c|c|c|c|c|c|c|c|}
\hline Series / $\mathrm{d}_{0}$ & 0.50 & 0.60 & 0.70 & 0.80 & 0.90 & 1.00 & 1.10 & 1.20 & 1.30 & 1.40 & 1.50 \\
\hline D1 & 20.89 & 15.09 & 9.69 & 5.22 & 1.84 & $\mathbf{- 0 . 5 8}$, & -2.31 & -3.54 & -4.45 & -5.14 & -5.68 \\
\hline D3 & 21.97 & 15.83 & 10.16 & 5.50 & 2.01 & $\mathbf{- 0 . 4 8}$, & -2.24 & -3.50 & -4.43 & -5.13 & -5.67 \\
\hline D5 & 22.06 & 15.90 & 10.23 & 5.57 & 2.05 & $\mathbf{- 0 . 4 5}$ & -2.23 & -3.51 & -4.44 & -5.15 & -5.70 \\
\hline D7 & 21.65 & 15.63 & 10.10 & 5.54 & 2.10 & $\mathbf{- 0 . 3 8}$, & -2.16 & -3.44 & -4.38 & -5.10 & -5.66 \\
\hline D10 & 21.23 & 15.38 & 9.97 & 5.50 & 2.10 & $\mathbf{- 0 . 3 7}$ & -2.14 & -3.43 & -4.38 & -5.10 & -5.66 \\
\hline \hline
\end{tabular}

ii) $\operatorname{AR}(1)$ disturbances

\begin{tabular}{|c|c|c|c|c|c|c|c|c|c|c|c|}
\hline Series / $\mathrm{d}_{0}$ & 0.50 & 0.60 & 0.70 & 0.80 & 0.90 & 1.00 & 1.10 & 1.20 & 1.30 & 1.40 & 1.50 \\
\hline D1 & 3.88 & 3.61 & 3.24 & 1.93 & $\mathbf{0 . 5 1}$ & $\mathbf{- 0 . 6 5}$ & $\mathbf{- 1 . 4 8}$ & -2.05 & -2.46 & -2.77 & -3.03 \\
\hline D3 & 9.05 & 8.35 & 5.18 & 2.52 & $\mathbf{0 . 6 5}$ & $\mathbf{- 0 . 6 5}$ & $\mathbf{- 1 . 5 9}$ & -2.29 & -2.83 & -3.28 & -3.66 \\
\hline D5 & 10.90 & 8.51 & 4.72 & 2.06 & $\mathbf{0 . 2 7}$ & $\mathbf{- 1 . 0 2}$ & -2.00 & -2.76 & -3.36 & -3.83 & -4.22 \\
\hline D7 & 11.03 & 7.65 & 3.81 & $\mathbf{1 . 4 0}$ & $\mathbf{- 0 . 1 6}$ & $\mathbf{- 1 . 3 4}$ & -2.28 & -3.03 & -3.63 & -4.11 & -4.50 \\
\hline D10 & 9.47 & 6.52 & 3.45 & $\mathbf{1 . 6 2}$ & $\mathbf{0 . 3 5}$ & $\mathbf{- 0 . 7 5}$ & -1.75 & -2.60 & -3.31 & -3.88 & -4.33 \\
\hline \hline
\end{tabular}

iii) $\operatorname{AR}(2)$ disturbances

\begin{tabular}{|c|c|c|c|c|c|c|c|c|c|c|c|}
\hline Series / $\mathrm{d}_{0}$ & 0.50 & 0.60 & 0.70 & 0.80 & 0.90 & 1.00 & 1.10 & 1.20 & 1.30 & 1.40 & 1.50 \\
\hline D1 & 3.11 & 2.82 & 2.73 & 1.71 & $\mathbf{0 . 3 2}$ & $\mathbf{- 0 . 9 6}$ & $\mathbf{- 1 . 4 9}$ & -2.67 & -3.14 & -3.46 & -3.69 \\
\hline D3 & 4.30 & 4.05 & 2.65 & 1.73 & $\mathbf{0 . 5 4}$ & $\mathbf{- 0 . 5 8}$ & $\mathbf{- 1 . 5 3}$ & -2.29 & -2.88 & -3.36 & -3.75 \\
\hline D5 & 3.58 & 3.03 & 2.66 & 2.03 & $\mathbf{1 . 0 3} \boldsymbol{\prime}^{\prime}$ & $\mathbf{- 0 . 0 6}$ & -1.69 & -1.97 & -2.71 & -3.31 & -3.82 \\
\hline D7 & 3.58 & 2.99 & 2.21 & $\mathbf{1 . 6 4}$ & $\mathbf{0 . 9 0}$ & $\mathbf{- 0 . 0 7}$ & -1.71 & -1.94 & -2.70 & -3.35 & -3.89 \\
\hline D10 & 2.64 & 1.95 & 1.84 & $\mathbf{1 . 6 1}$ & $\mathbf{1 . 1 9}$ & $\mathbf{0 . 4 0}$ & -1.67 & -1.99 & -2.34 & -3.09 & -3.72 \\
\hline \hline
\end{tabular}

' and in bold: Non-rejection values at the 5\% significance level. 
TABLE 2

Values of $\mathrm{d}_{0}$ which produce the lowest $|\hat{r}|$ for each series

\begin{tabular}{|c|c|c|c|c|c|}
\hline $\mathrm{u}_{\mathrm{t}} /$ Series & $\mathrm{D} 1$ & $\mathrm{D} 3$ & $\mathrm{D} 5$ & $\mathrm{D} 7$ & $\mathrm{D} 10$ \\
\hline White nosie & 0.97 & 0.98 & 0.98 & 0.98 & 0.98 \\
\hline AR (1) & 0.91 & 0.94 & 0.91 & 0.88 & 0.93 \\
\hline AR (2) & 0.92 & 0.95 & 1.00 & 1.00 & 1.04 \\
\hline
\end{tabular}

TABLE 3

Diagnostic tests on the residuals of the selected models

\begin{tabular}{|c|c|c|c|}
\hline Series & & Model & Diagnostics \\
\hline \multirow{4}{*}{ D1 } & A) & $(1-L)^{0.97} x_{t}=\varepsilon_{t}$ & B \\
\cline { 2 - 4 } & B) & $(1-L)^{0.91} x_{t}=u_{t}, u_{t}=0.38 u_{t-1}$ & A B C \\
\hline & C) & $(1-L)^{0.92} x_{t}=u_{t}, u_{t}=0.37 u_{t-1}-0.07 u_{t-2}$ & A B C \\
\hline \hline \multirow{5}{*}{ D3 } & A) & $(1-L)^{0.98} x_{t}=\varepsilon_{t}$ & B C \\
\hline & B) & $(1-L)^{0.94} x_{t}=u_{t}, u_{t}=0.14 u_{t-1}$ & A B C \\
\hline & C) & $(1-L)^{0.95} x_{t}=u_{t}, u_{t}=0.13 u_{t-1}-0.01 u_{t-2}$ & A B \\
\hline \hline \multirow{5}{*}{ D5 } & A) & $(1-L)^{0.98} x_{t}=\varepsilon_{t}$ & B \\
& B) & $(1-L)^{0.91} x_{t}=u_{t}, u_{t}=0.10 u_{t-1}$ & A B C \\
\hline & C) & $(1-L) x_{t}=u_{t}, u_{t}=0.01 u_{t-1}-0.10 u_{t-2}$ & A B \\
\hline \hline \multirow{5}{*}{ D7 } & A) & $(1-L)^{0.98} x_{t}=\varepsilon_{t}$ & A B C \\
\hline & B) & $(1-L)^{0.88} x_{t}=u_{t}, u_{t}=0.08 u_{t-1}$ & A B \\
\hline & C) & $(1-L) x_{t}=u_{t}, u_{t}=-0.03 u_{t-1}-0.13 u_{t-2}$ & A B \\
\hline \hline \multirow{5}{*}{ D10 } & A) & $(1-L)^{0.98} x_{t}=\varepsilon_{t}$ & A B C \\
\hline & B) & $(1-L)^{0.93} x_{t}=u_{t}, u_{t}=0.08 u_{t-1}$ & A B \\
\hline & C) & $(1-L)^{1.04} x_{t}=u_{t}, u_{t}=-0.06 u_{t-1}-0.12 u_{t-2}$ & A B \\
\hline
\end{tabular}

N.B. A: no serial correlation; B: homoscedasticity; C: functional form at the 95\% significance level. 


\begin{tabular}{|c|c|c|c|c|}
\hline \multicolumn{5}{|c|}{ TABLE 4} \\
\hline \multicolumn{5}{|c|}{ Impulse responses of the selected models } \\
\hline & D1 & D3 & D5 & $\mathrm{D} 7$ and $\mathrm{D} 10$ \\
\hline Selected model & $\mathrm{B}$ & $\mathrm{B}$ & $\mathrm{B}$ & A \\
\hline 0 & 1.000 & 1.000 & 1.000 & 1.000 \\
\hline 1 & 1.290 & 1.079 & 1.010 & 0.980 \\
\hline 2 & 1.359 & 1.062 & 0.970 & 0.970 \\
\hline 3 & 1.359 & 1.042 & 0.939 & 0.963 \\
\hline 4 & 1.340 & 1.026 & 0.918 & 0.958 \\
\hline 5 & 1.318 & 1.013 & 0.900 & 0.955 \\
\hline 6 & 1.298 & 1.002 & 0.887 & 0.951 \\
\hline 7 & 1.280 & 0.993 & 0.875 & 0.949 \\
\hline 8 & 1.264 & 0.986 & 0.865 & 0.946 \\
\hline 9 & 1.250 & 0.979 & 0.856 & 0.944 \\
\hline 10 & 1.238 & 0.973 & 0.848 & 0.942 \\
\hline 20 & 1.162 & 0.934 & 0.798 & 0.930 \\
\hline 30 & 1.120 & 0.912 & 0.770 & 0.922 \\
\hline 40 & 1.091 & 0.897 & 0.751 & 0.917 \\
\hline 50 & 1.069 & 0.885 & 0.736 & 0.913 \\
\hline 60 & 1.052 & 0.875 & 0.724 & 0.910 \\
\hline 70 & 1.037 & 0.867 & 0.714 & 0.907 \\
\hline 80 & 1.025 & 0.860 & 0.705 & 0.905 \\
\hline 90 & 1.014 & 0.854 & 0.698 & 0.903 \\
\hline 100 & 1.004 & 0.849 & 0.691 & 0.901 \\
\hline 125 & 0.984 & 0.838 & 0.678 & 0.897 \\
\hline 150 & 0.968 & 0.828 & 0.667 & 0.893 \\
\hline 175 & 0.955 & 0.821 & 0.657 & 0.891 \\
\hline 200 & 0.943 & 0.814 & 0.650 & 0.888 \\
\hline
\end{tabular}

